\title{
Computation of Multiple Inverse Kinematic Solutions for Redundant Manipulators by Inverting Modular Neural Networks
}

\author{
Non-member Bao-Liang Lu (RILEN) \\ Member Koji Ito (Toyohashi Univ. of Tech.; RIKEN)
}

\begin{abstract}
This paper presents a new approach to computing multiple inverse kinematic solutions for redundant manipulators by inverting modular neural networks. This approach is a three-phase procedure. In the first phase, the configuration space is partitioned into a set of regions to learn the forward kinematic function of a manipulator, and a set of modular neural networks is trained on associated sets of input-output data sampled over these regions. In the second phase, multiple inverse kinematic solutions for a desired endeffector position are computed by inverting the corresponding trained modular neural networks. In the third phase, an "optimal" solution is selected from the multiple inverse kinematic solutions according to a given criterion. The important advantage of this approach over existing methods is that an "optimal" inverse kinematic solution can be obtained from the multiple solutions. Therefore, better control of the manipulator can be achieved. This approach is illustrated with a three-joint planar arm.
\end{abstract}

Key words: Inverse kinematics, Modular neural network, Separable programming, Network inversion.

\section{Introduction}

The forward kinematic function for a robot manipulator is a nonlinear mapping,

$$
h: Q \subseteq \mathbf{R}^{n} \rightarrow \boldsymbol{P} \subseteq \mathbf{R}^{m},
$$

which maps a set of joint-angle variables from the configuration space, $\boldsymbol{Q}$, to the workspace, $\boldsymbol{P}$, where $\mathbf{R}^{n}$ and $\mathbf{R}^{m}$ are the $n$-dimensional and the $m$-dimensional spaces, respectively. When $m<n$, the manipulator is called a redundant manipulator. The inverse kinematics problem for redundant manipulators is to find some joint-angle values $q \in \boldsymbol{Q}$ such that $\boldsymbol{h}(\boldsymbol{q})$ is a desired end-effector position $\boldsymbol{p} \in \boldsymbol{P}$. This problem is an ill-posed problem because the inverse kinematic mapping,

$$
\boldsymbol{h}^{-1}: \boldsymbol{P} \subseteq \mathbf{R}^{m} \rightarrow \boldsymbol{Q} \subseteq \mathbf{R}^{n}
$$

is an one-to-many mapping. In general, this problem is locally ill-posed in the sense that it has no unique solution and globally ill-posed because there are mul- tiple solution branches. Hence, there is no closed-form direct expression for the inverse linematic mapping.

In the last few years, sevcral approaches to solving the inverse kinematics problem using neural net works have been proposed ${ }^{(1)-(5)}$. Two popular mothods are the direct inverse approach and the indirect inverse approach. The direct inverse approach (1) learns the inverse kinematic mapping directly using supervised learning algorithms. This approach suffers two main drawbacks that limit its usefulness. First, when the inverse images are nonconvex, the inverse kimematic solution can not be obtained by this approach because any supervised learning algorithm is unable to leam an one-to-many mapping. Second, the multiple solution branch problem is not considered, and hence, for a clesired end-effector position $\boldsymbol{p}$ only one inverse solution $\boldsymbol{q}$ can be found. The indirect inverse approach ${ }^{(4)}$ is a two-phase procedure. In the first phase, a network (Net. $f$ ) is trained to approximate the forward kinematic mapping, $\boldsymbol{Q} \rightarrow \boldsymbol{P}$. After the training is 
completed, all the parameters of Net $_{f}$ are fixed. In the second phase, a particular inverse solution is obtained by placing another network ( Net $_{i}$ ) and Net $f$ in series and learning an identity mapping across the composite network formed by Net; and Net $f$. Although the indirect inverse approach can overcome the nonconvex problem encounted by the direct inverse approach, the multiple solution branch problem is still unsolved.

This paper presents a new approach to solving the inverse kinematics problem for reclundant manipulators. This approach is a three-phase procedure. In the first phase, the configuration space is partitioned into a set of regions and a set of modular neural networks is trained to learn the forward kinematic function of a manipulator. In the second phase, the multiple inverse kinematic solutions for a desired end-effector position are computed by inverting the corresponding modular neural networks. In the third phase, an "optimal" solution is selected from the multiple solutions according to a given criterion. Since the inverse kinematic solutions are obtained by inverting the corresponding modular networks, there is no nonconvex problem in this approach. The important advantage of this approach over existing methods is that an "optimal" inverse kinematic solution can be obtained from the multiple solutions. As a result, better control of the manipulator using the "optimal" inverse solution than that using an ordinary inverse solution in existing methods can be achieved.

\section{The Proposed Approach}

Let us consider a redundant robot manipulator. We assume that a sufficiently large number of inputontput training data can be gathered by sampling the configuration space and identifying the associated points in the work space.

\subsection{Learning by Modular Networks}

The forward kinematic function can be approximated by a monolithic multilayer neural network. This strategy is commonly used for approximation of inverse kinematics in the robotics literature ${ }^{(1)-(2)}$. However, the monolithic structure has two drawbacks: (a) to approximate the forward kinematic function of a manipulator with large excess degrees of freedom requires long training time from the point of view of learning a function, and (b) to invert the network becomes difficult because the large-scale network increases complexity of the inversion problem from the point of view of inverting a network. In order to overcome the above disadvantages, a modular network scheme is introduced in this paper.

To implement the modular scheme, we need to decompose the learning task into subtasks which can be learned by individual modular networks. For the forward kinematic function approximation problem, this can be achieved by partitioning the configuration space into a set of regions. We assume that the configuration space is a convex polyhedron ${ }^{(6)}$ in $\mathbf{R}^{\prime \prime}$. For the simplest special case, it can be expressed as the following form:

$$
\boldsymbol{Q} \equiv\left\{\boldsymbol{q} \in \mathbf{R}^{n} \mid \boldsymbol{q}^{\min } \leq \boldsymbol{q} \leq \boldsymbol{q}^{\text {max }}\right\}
$$

where $\boldsymbol{q}^{\text {min }}$ and $\boldsymbol{q}^{\max }$ are constant vectors and represent lower and upper joint-angle limits, respectively. In this case, the configuration space $\boldsymbol{Q}$ can be easily partitioned into $\tau(\tau \geq 1)$ smaller convex polyhedrons as follows:

$$
\begin{gathered}
\boldsymbol{Q}_{i} \equiv\left\{q_{i} \in \mathbf{R}^{n} \mid \boldsymbol{q}_{i}^{\min } \leq \boldsymbol{q}_{i} \leq \boldsymbol{q}_{i}^{\text {max }}\right\} \\
\text { for } i=1,2, \cdots, \tau
\end{gathered}
$$

where $\boldsymbol{q}_{i}{ }^{\text {min }}$ and $\boldsymbol{q}_{i}{ }^{\max }$ are also constant vectors and represent lower and upper joint-angle limits in the $i$ th region $\boldsymbol{Q}_{i}$, respectively.

If the input-output training clata sets $T_{1}, T_{2}, \cdots$, $T_{+}$are sampled over the corresponding disjoint regions $\boldsymbol{Q}_{1}, \boldsymbol{Q}_{2}, \cdots, \boldsymbol{Q}_{\boldsymbol{\tau}}$, the accuracy of approximation on the boundary of each region may become bad because there is no any training clata outside the boundary of each region. In order to deal with this problem, the training data sets $T_{1}, T_{2}, \cdots, T_{T}$ are sampled over the corresponding overlapping regions $\hat{\boldsymbol{Q}}_{1}, \hat{\boldsymbol{Q}}_{2}, \cdots, \hat{\boldsymbol{Q}}_{\boldsymbol{T}}$. The $\hat{Q}_{i}$ is defined as follows:

$$
\begin{aligned}
\hat{\boldsymbol{Q}}_{i} \equiv & \left\{\boldsymbol{q}_{i} \in \mathbf{R}^{n} \mid \hat{\boldsymbol{q}}_{i}^{\min } \leq \boldsymbol{q}_{i} \leq \hat{\boldsymbol{q}}_{i}^{\max }\right\}, \\
& \text { for } i=1,2, \cdots, \tau,
\end{aligned}
$$

where $\hat{\boldsymbol{q}}_{\boldsymbol{i}}^{\text {min }}<\boldsymbol{q}_{i}^{\text {min }}$ and $\boldsymbol{q}_{i}^{\text {max }}<\hat{\boldsymbol{q}}_{i}^{\max }$.

Based on the above discussion, the forward linematic function can be learned by individual modular neural networks according to the following algorithm:

Step 1 : Partition the configuration space $\boldsymbol{Q}$ into $\tau$ regions $\boldsymbol{Q}_{1}, \boldsymbol{Q}_{2}, \cdots, \boldsymbol{Q}_{\boldsymbol{\tau}}$ in a uniform grid or in a non-uniform grid.

Step 2: Gather the input-output training data sets $T_{1}, T_{2}, \cdots, T_{r}$ by sampling the corresponding 
overlapping regions $\hat{\boldsymbol{Q}}_{1}, \hat{\boldsymbol{Q}}_{2}, \cdots, \hat{\boldsymbol{Q}}_{r}$, and iclentifying the associated points in the workspace.

Step 3: Memorize the ranges of unnormalized desired outputs $^{1}$ in $T_{1}, T_{2}, \cdots, T_{\tau}$, in order to select modular networks for inverting.

Step 4: Train the modular neural network $M N_{1}$, $M N_{2}, \cdots, M N_{\tau}$ on $T_{1}, T_{2}, \cdots, T_{\tau}$, respectively.

It is important to emphasize that, besides overcoming the drawbacks of the monolithic structure mentioned above, the modular network scheme can regularize the ill-posed inverse kinematics problem globally to certain extent. Although there is no theoretical guarantee that all multiple solution branches can be partitioned by dividing the configuration space into a set of regions, typically, the smaller the region is divided, the more complete the global regularization can be achieved. Figure 1 shows the modular network scheme for learning the forward kinematic function.

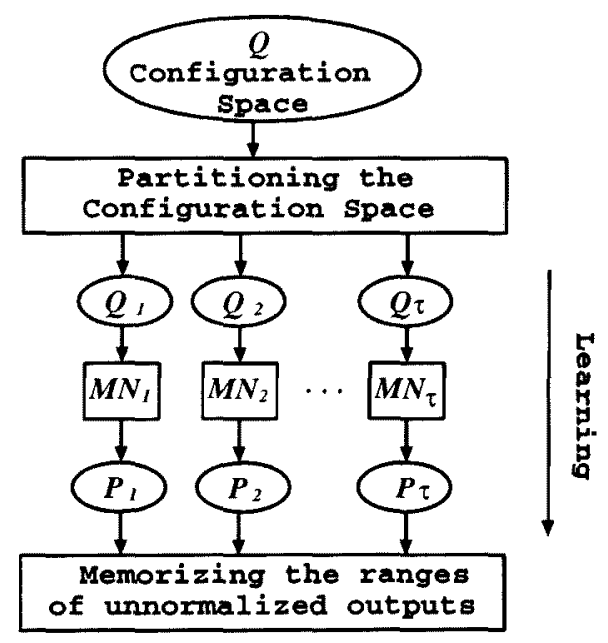

Figure 1: The modular network scheme for leaning the forward kinematic function.

\subsection{Network Inversion}

The inversion problem for multilayer networks is to find inputs which yield a desired output. There are several approaches to inverting multilayer networks in

\footnotetext{
${ }^{1}$ Since all of the training input and output data are normalized between 0 and 1 before training, we can only distinguish the output ranges by using unnormalized data after training.
}

the neural network literature ${ }^{(7)-(11)}$. In this paper, the optimization inversion approach ${ }^{(9)-(10)}$ is used.

In the optimization inversion approach, the inversion problem is formulated as a separable nonlinear programming problem ${ }^{(12)-(13)}$ as follows ${ }^{2}$ :

$$
\begin{array}{ll}
\text { Minimize } & g\left(\boldsymbol{x}_{1}\right) \\
\text { Subject to } & W_{3} f_{2}\left(\tilde{\boldsymbol{b}}_{2}\right)=\tilde{\boldsymbol{b}}_{3}-\text { bias }_{3} \\
& W_{2} \boldsymbol{x}_{1}-\tilde{\boldsymbol{b}}_{2}=- \text { bias }_{2} \\
& \Gamma \leq \boldsymbol{x}_{1} \leq \Theta
\end{array}
$$

where $W_{3}, W_{2}$, bias $_{3}$, bias $s_{2}, \tilde{b}_{3}, \Gamma$, and $\Theta$ are given, $\boldsymbol{x}_{1}$ and $\tilde{b}_{2}$ are unknown vectors, $\boldsymbol{x}_{1}=\left[x_{11}, x_{12}, \cdots\right.$, $\left.x_{1 N_{1}}\right]^{T} \in \mathbf{R}^{N_{1}}, W_{r}=\left[\boldsymbol{w}_{r 1}, \boldsymbol{w}_{r_{2}}, \cdots, \boldsymbol{w}_{r N_{r}}\right]^{T} \in \mathbf{R}^{N_{r} \times N_{r-1}}$ $, \boldsymbol{w}_{r j}=\left[w_{r j 1}, w_{r j 2}, \cdots, w_{r j N_{r-1}}\right], j=1,2, \cdots, N_{r}$, $f_{r}=\left[f_{r 1}, f_{r 2}, \cdots, f_{r_{r}}\right]^{T}$, bias $_{r}=\left[\right.$ bias $s_{r 1}$, bias $_{r 2}$, $\cdots$, bias $\left._{r N_{r}}\right]^{T} \in \mathbf{R}^{N_{r}}, \boldsymbol{b}_{r}=\left[\begin{array}{ll}b_{r 1}, & b_{r^{\prime}}, \cdots, b_{r} N_{r}\end{array}\right]^{T} \in$ $\mathbf{R}^{N_{r}}, \tilde{b}_{r}=b_{r}+$ bias $_{r}, r=2,3, \Gamma=\left[\gamma_{1}, \gamma_{2}, \cdots, \gamma_{N_{1}}\right]^{T}$, $\Theta=\left[\theta_{1}, \theta_{2}, \cdots, \theta_{N_{1}}\right]^{T}, \tilde{\boldsymbol{b}}_{3}=\overline{\boldsymbol{b}}_{3}+$ bias $_{3}=\boldsymbol{f}_{3}^{-1}\left(\overline{\boldsymbol{x}}_{3}\right)$, $\boldsymbol{f}_{3}^{-1}\left(\overline{\boldsymbol{x}}_{3}\right)=\left[f_{31}^{-1}\left(\bar{x}_{31}\right), f_{32}^{-1}\left(\bar{r}_{32}\right), \cdots, f_{3 N_{3}}^{-1}\left(\bar{r}_{3 N_{3}}\right)\right]^{T}$.

The introduction of $\Gamma \leq x_{1} \leq \Theta$ into Eq. (6) is to limit the values of obtained inversions within meaningful ranges. The objective function $g\left(x_{1}\right)$ can take a form: $g\left(x_{1}\right)= \pm x_{1 l}$ for $l=1,2, \cdots$, or $N_{1} ; g\left(x_{1}\right)=$ $\left\|\boldsymbol{x}_{1}-\boldsymbol{c}\right\|^{2}$, where $c=\left[c_{1}, c_{2}, \cdots, c_{N_{1}}\right]^{T} \in \mathbf{R}^{N_{1}}$ is a given reference point in the input space; or any one of other optimization criteria. An important advantage of the optimization inversion approach is that the inverse problem defined by Eq. (6) can be approximated by a pseudo linear programming problem and solved by a variation of the simplex method ${ }^{(12)-(13)}$, a common and efficient technique for solving linear programning problems.

\subsection{Multiple Inverse Solutions}

If a forward kinematic function is approximated precisely by a multilayer network, then solving the inverse kinematics problem is equivalent to inverting the multilayer network. Suppose a desired end-effector position $\boldsymbol{p}$ is a point within the worlispace $\boldsymbol{P}$. To compute the inverse kinematic solutions associated

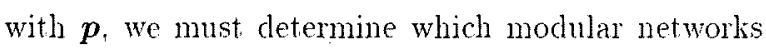
need to be inverted. However, it is difficult for us to make a precise choice because, in general, $\boldsymbol{P}_{1}$, $\boldsymbol{P}_{2}, \cdots, \boldsymbol{P}_{\tau}$, the images of $\boldsymbol{Q}_{1}, \boldsymbol{Q}_{2}, \cdots, \boldsymbol{Q}_{\tau}$, are non-convex sets and there is no closed-form expression. In this paper, we use a simple approach to tackling this problem, that is, whether $\boldsymbol{p}$ is within $\boldsymbol{P}_{i}$ is

\footnotetext{
${ }^{2}$ The notation is provided in Appendix
} 
judged according to the range of unnormalized training outputs in $T_{i}$. For example, if $\boldsymbol{p}=(0.65,0.0)$ and the ranges of unnormalized training outputs in $T_{1}$ and $T_{2}$ are $\{(0.01,0.70),(-0.40,0.60)\}$ and $\{(-0.55,0.53),(0.16,0.70)\}$, respectively (see Table 1 ), then only $M N_{1}$ is selected because $\boldsymbol{p}$ is within the range of $T_{1}$, while $\boldsymbol{p}$ is not included in the range of $T_{2}$. This is an approximate approach, and therefore, more number of the modular networks than those actually required may be selected and there may exist no inverse solution for some modular networks that are selected incorrectly. For a desired end-effector position $\boldsymbol{p}, \sigma(1 \leq \sigma \leq \tau)$ modular networks may be selected for inverting because some of $\boldsymbol{P}_{1}, \boldsymbol{P}_{2}, \cdots$, $\boldsymbol{P}_{\tau}$ overlap each other.

The goal of the proposed approach is to find different inverse linematic solutions $q$ as many as possible for a desired end-effector position $p$. The multiple solutions to the inverse kinematics problem can be obtained by the following algorithm:

Step 1: Determine which modular network needs to be inverted.

Step 2: Select objective fuuction $g(\boldsymbol{q})^{3}$ in Eq. (6).

Step 3: Compute an inverse kinematic solution by solving the separable nonlinear programming problem defined by Eq. (6).

Step 4: Repeat Steps 2 and 3 until a desired number of inverse solutions are obtained.

Figure 2 illustrates the scheme for computing multiple inverse kinematic solutions by inverting modular neural networks.

\subsection{Optimum Inverse Solution}

After the multiple inverse solutions have been obtained, we must select an "optimum" one from them. The "optimum" inverse solution refers to the best joint angle $q$ in obtained multiple inverse kinematic solutions for a desired end-effector position $\boldsymbol{p}$. The criterion for choosing an "optimum" inverse solution is

\footnotetext{
${ }^{3}$ Note that for following the conventional notation in the robotics and neural networks literature, we use two sets of symbols for describing the inverse kinematics problem and neural network inversions, respectively. The relationships among some of the major symbols are as follows: $q \equiv x_{1}, \boldsymbol{p} \equiv x_{3}, n \equiv N_{1}$, and $m \equiv N_{3}$.
}

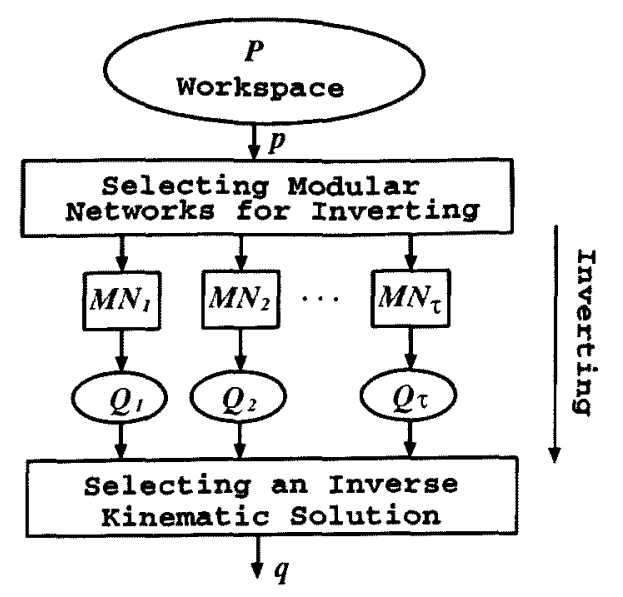

Figure 2: Illustration of computing multiple inverse kinematic solutions by inverting the modular neural networks.

largely dependent on the requirement of a manipulator, and it is difficult to give a general criterion. We will discuss how to choose an optimum inverse solution through a simple example.

Consider, for example, a three-joint planar arm as shown in Figure 3 . The workspace contains an obstacle. If the manipulator needs to move from position $\boldsymbol{p}^{\prime}$ to position $\boldsymbol{p}$, we must find the corresponding q. According to $\mathrm{Eq}_{\mathrm{l}}$. (6), we can obtain many inverse kinematic solutions by setting the objective function in the forms: Minimize $q_{i}$ or Maximize $q_{i}$ for $i=1,2$, or 3 . For example, two different inverse solutions are illustrated in Figure 3 as the elbow-up and elbow-down solutions. In this case, the movement may not be free of collisions between the manipulator and the obstacle. In order to avoid the obstacle we prefer to select the elbow-up solution as an "optimal" one since the elbow. up solution reduces the chance of a collision between the links of the manipulator and the obstacle resting on the workspace.

\section{Simulation Results}

In orcler to clemonstrate the proposed approach, the simulations are carried out on a three-joint planar arm as shown in Figure 4. The configuration of the arm is characterized by the three joint angles, $q_{1}, q_{2}$, and $q_{3}$ and the corresponding pair of Cartesian variables $p_{1}$ and $p_{2}$. Without loss of generality and for simplic- 


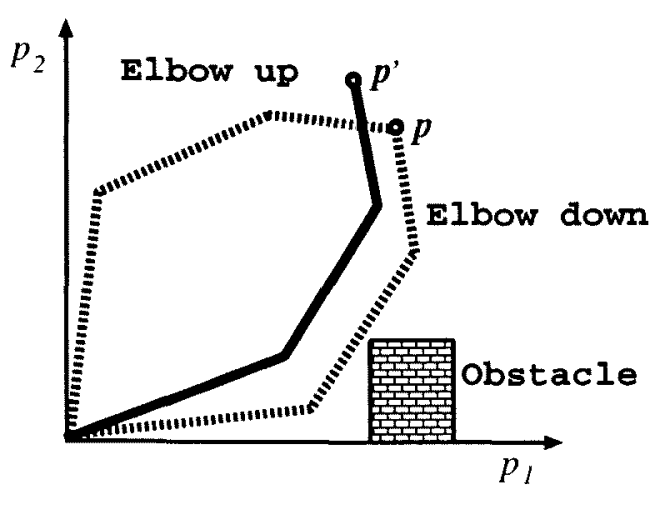

Figure 3: Two different inverse kinematic solutions for a redundant manipulator.

ity of illustration, the precise analytic forward kinematic function of the arm is used for generating training imput-output data. It is expressed as follows:

$$
\begin{aligned}
p_{1}= & L_{1} \cos \left(q_{1}\right)+L_{2} \cos \left(q_{1}+q_{2}\right)+ \\
& L_{3} \cos \left(q_{1}+q_{2}+q_{3}\right) \\
p_{2}= & L_{1} \sin \left(q_{1}\right)+L_{2} \sin \left(q_{1}+q_{2}\right)+ \\
& L_{3} \sin \left(q_{1}+q_{2}+q_{3}\right)
\end{aligned}
$$

where $L_{1}, L_{2}$, and $L_{3}$ are the manipulator link lengths. We set $L_{1}=0.3, L_{2}=0.25$, and $L_{3}=0.15$, and restrict the motion of the joints $q_{1}, q_{2}$, and $q_{3}$ to the intervals $[-\pi / 6,2 \pi / 3],[0,5 \pi / 0]$, and $[-\pi / 6, \pi / 6]$, respectively.

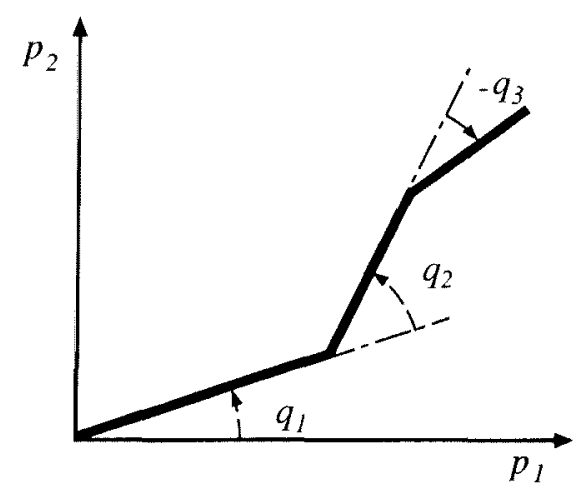

Figure 4: A three-joint planar arm.

The configuration space $\boldsymbol{Q}$ is divided into 8 overlapping regions $\hat{\boldsymbol{Q}}_{1}, \hat{\boldsymbol{Q}}_{2}, \cdots, \hat{\boldsymbol{Q}}_{8}$, via the grid points $(-\pi / 6, \quad 3 \pi / 12,2 \pi / 3), \quad(0, \quad 5 \pi / 12, \quad 5 \pi / 6)$, and $(-\pi / 6,0, \pi / 6)$. For example, $\hat{\boldsymbol{Q}}_{1}$ is partitioned by the
Table 1: The ranges of unnormalized outputs in $T$, for $i=1,2, \cdots, 8$

\begin{tabular}{lllllllll} 
& $T_{1}$ & $T_{2}$ & $T_{3}$ & $T_{4}$ & $T_{5}$ & $T_{6}$ & $T_{7}$ & $T_{8}$ \\
\hline$p_{1}^{\min }$ & .01 & -.55 & -.20 & -.56 & -.06 & -.57 & -.19 & -.58 \\
$p_{1}^{\max }$ & .70 & .53 & .61 & .18 & .70 & .53 & .59 & .02 \\
$p_{2}^{\min }$ & -.40 & .16 & .13 & -.16 & -.35 & .11 & .04 & -.17 \\
$p_{2}^{\max }$ & .60 & .70 & .57 & .58 & .60 & .60 & .52 & .59 \\
\hline
\end{tabular}

intervals $[-\pi / 6,3 \pi / 12],[0,5 \pi / 12]$, and $[-\pi / 6,0]$. Over each of the regions, a set of $216(6 \times 6 \times 6)$ training input-output data is sampled using Eq. (T) in a non-iniform grid. Table 1 shows the ranges of unnormalized outputs of $T_{1}, T_{2}, \cdots, T_{8}$, respectively. Eight modular networks are used for approximating the forward kinematic function. Each of them is a threelayer netwolk with 3 input, 10 hidden and 2 output units, and is trained by the backpropagation learning algorithm $^{(1+1)}$.

To compute multiple inverse kincmatic solutions, we select the objective function $g(q)$ in $\mathrm{Eq}$. (6) as: $g(\boldsymbol{q})= \pm q$ for $i=1,2$, or 3 . This objective function allows us to minimize or maximize the movement of the ith link. Let us compute the inverse kinematic solutions for the desired end-effector position $\boldsymbol{p}=(0.65,0.0)$. From Table 1 , we see that the desired end-effector position $p$ is located in the ranges of the training outputs of $T_{1}$ and $T_{5}$. Therefore, $M N_{1}$ and $M N_{\text {5 }}$ are selceted for inverting. Solving the separable nonlincar programming problem defined by Eq. (6) by use of the simplex method with the restricted basis entry rulc(12), we obtain twelve inverse linematic solutions (sec Table 2). Among them only four inverse solutions are distinct from each other because the excess degree of frecdom in the inverse kinematic problem is only one. Figure 5 illustrates these four different inverse solutions in the workspace.

In the similar way, the inverse kinematic solutions for the desired end-effector position $\boldsymbol{p}=(-0.4,0.2)$ are computed. In this case, $M N_{2}, M N_{4}, M N_{6}$, and $M N_{8}$ are selected for inverting. However, only four distinct inverse solutions, which are shown in Table 3 and illustrated in Figure 4, are obtained by inverting $M N_{4}$ and $M N_{8}$, and there exists no any inverse solution in $M N_{2}$ and $M N_{6}$. The reason for this situation is that $\boldsymbol{p}=(-0.4,0.2)$ is within the training output ranges of $T_{2}$ and $T_{6}$, but it doesn't locate in the actual areas of the training out puts of $T_{2}$ and $T_{6}$. 
Table 2: The Inverse Iinematic Solutions for $p_{1}=$ 0.65 and $p_{2}=0.00$, and the Corresponding Actual Positions

\begin{tabular}{cllllll} 
No. & \multicolumn{4}{c}{ Inverse Kinematic Solutions $\dagger$} & \multicolumn{2}{c}{ Positions } \\
\hline & & $q_{1}$ & $q_{2}$ & $q_{3}$ & $p_{1}$ & $p_{2}$ \\
\hline \multirow{4}{*}{$M N_{1}$} & $\operatorname{Min}\left(q_{1}\right)$ & -23.8 & 42.0 & 0.0 & 0.655 & 0.004 \\
& $\operatorname{Max}\left(q_{1}\right)$ & -20.9 & 48.1 & -29.9 & 0.652 & 0.000 \\
& $\operatorname{Man}\left(q_{2}\right)$ & -23.8 & 42.0 & 0.0 & 0.655 & 0.004 \\
& $\operatorname{Max}\left(q_{2}\right)$ & -20.9 & 48.1 & -29.9 & 0.652 & 0.000 \\
& $\operatorname{Min}\left(q_{3}\right)$ & -20.9 & 48.1 & -29.9 & 0.652 & 0.000 \\
& $\operatorname{Max}\left(q_{3}\right)$ & -23.8 & 42.0 & 0.0 & 0.655 & 0.004 \\
\hline \multirow{4}{*}{$M N_{5}$} & $\operatorname{Min}\left(q_{1}\right)$ & -25.1 & 43.1 & 0.0 & 0.652 & -0.003 \\
& $\operatorname{Max}\left(q_{1}\right)$ & -22.3 & 27.3 & 29.9 & 0.650 & -0.005 \\
& $\operatorname{Min}\left(q_{2}\right)$ & -22.3 & 27.3 & 29.9 & 0.650 & -0.005 \\
& $\operatorname{Max}\left(q_{2}\right)$ & -25.1 & 43.1 & 0.0 & 0.652 & -0.003 \\
& $\operatorname{Min}\left(q_{3}\right)$ & -25.1 & 43.1 & 0.0 & 0.652 & -0.003 \\
& $\operatorname{Max}\left(q_{3}\right)$ & -22.3 & 27.3 & 29.9 & 0.650 & -0.005 \\
\hline
\end{tabular}

$\dagger$ Note that the values of $q_{i}$ are in degrees.

Table 3: The Inverse Kinematic Solutions for $p_{1}=$ -0.4 and $p_{2}=0.2$, and the Corresponding Actual Positions

No. Inverse Kinematic Solutions Positions

\begin{tabular}{lllllll}
\hline & & $q_{1}$ & $q_{2}$ & $q_{3}$ & $p_{1}$ & $p_{2}$ \\
\hline$M N_{4}$ & $\operatorname{Min}\left(q_{1}\right)$ & 92.3 & 101.6 & 0.0 & -0.400 & 0.204 \\
& $\operatorname{Max}\left(q_{1}\right)$ & 95.5 & 110.7 & -29.9 & -0.403 & 0.198 \\
\hline$M N_{8}$ & $\operatorname{Min}\left(q_{1}\right)$ & 93.4 & 100.9 & 0.0 & -0.405 & 0.201 \\
& $\operatorname{Max}\left(q_{1}\right)$ & 96.0 & 87.9 & 29.9 & -0.405 & 0.198 \\
\hline
\end{tabular}

From the above simulation results, we see that the inverse kinematic solutions in multiple solution branches can be found by inverting the corresponding modular networks. This demonstrates that our approach can regularize the inverse kinematics problem globally, and furthermore, distinct solutions in the same solution branch can also be obtained by solving the optimization problem clefined in Eq. (6) with different objective functions.

After the multiple inverse linematic solution have been obtained, we can select an optimum solution from them according to requirement of the manipulator. For example, in order to avoid the obstacle resting on the workspace, the elbow-up solution $q=(96.0,87.9,29.9)$ as shown in Figure 6 is selected as an optimum solution from four inverse solutions as shown in Table 3.

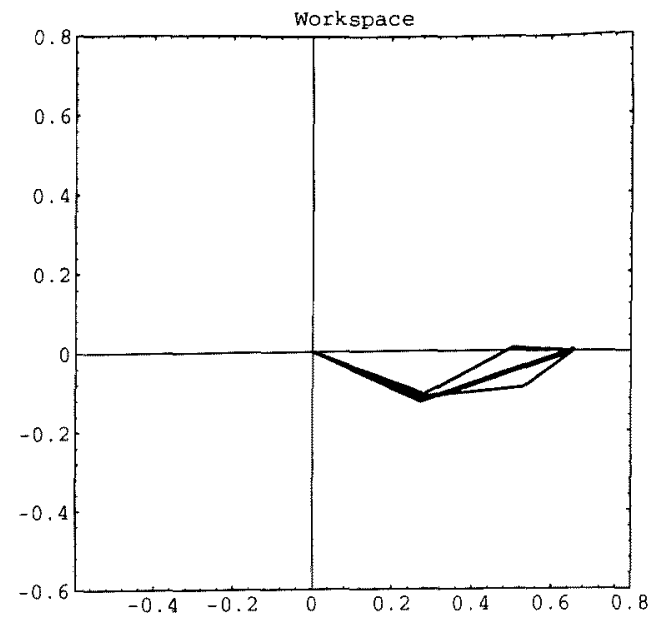

Figure 5: Four differcnt inverse linematic solutions obtained by inverting $M N_{1}$ and $M N_{5}$ for the desired end-effector position $p_{1}=0.65$ and $p_{2}=0.0$. Note that two of solutions are quite near and almost overlapped in the figure.

\section{Conclusion}

In this paper, we have presented a new approach to solving the inverse kinematics problem for reclundant manipulators. This approach is based on modular neural network scheme and network inversion techniques, This approach has an important feature in comparison with existing methods, that is, both the inverse kinematic solutions located in multiple solution branches and ones that belong to the same solution branch can be found by inverting the corresponding modular neural networks. Therefore, an optimum inverse kinematic solution can be found and better control of the manipulator can be achicved. As future work we will develop efficient approach to selecting modular networks for inverting and perform simulations on manipulators with large excess degrees of frecdom.

(Manuscript received 27 April 1995; revised 19 July 1995) 


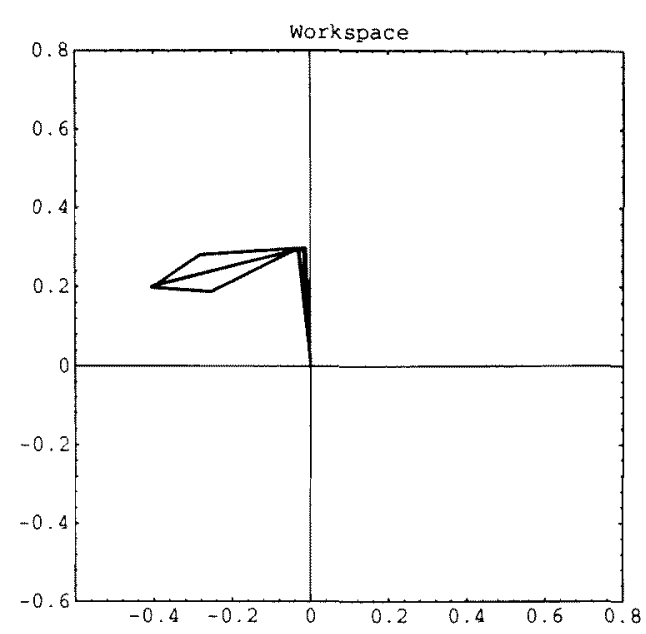

Figure 6: Four different inverse kinematic solutions obtained by inverting $M N_{4}$ and $M N_{8}$ for the desired end-effector position $p_{1}=-0.4$ and $p_{2}=0.2$. Note that two of solutions are quite near and overlapped in the figure.

\section{References}

(1) M. Kuperstein: "Adaptive visual-motor coordination in multijoint robots using parallel architecture", Proc. of 1987 IEEE Int. Conf. Robotics and Automation, p1). 1595-1602 (1987)

(2) H. J. Ritter, T. M. Martinetz \& K. J. Schulten : "Topology-conserring maps for lcarning visuomotor-coordination", Neural Netuorks, vol. 2, pp. $159-168$ (1989)

(3) M. Kawato: "Computational schemes and neural network models for formation and control of multijoint arm trajectory", in W. T. Miller, R. S. Sutton, \& P. J. Werbos (Eds.), Neural networks for Control, pp. 197-228 (1990)

(4) M. I. Jordan \& D. E. Rumelhart: "Forward models: supervised learning with a distal teacher", Cognitive Science, vol. 16, pp. 307-354 (1992)

(5) D. E. Demers: "Learning to invert many-to-one mapping", Ph. D. thesis, University of California, San Diego (1992)

(6) R. J. Schilling: Fundamentals of Robotics: Analysis and Control, Prentice Hall (1994)
(7) R. J. Williams : "Inverting a conncetionist network mapping by backpropagation of error". Proc. of 8th Annual Conference of th Cognitive Science Society, pp. 859-865 (1986)

(8) A. Linden \& J. Kindermann: "Inversion of Multilayer Nets", Proc. of International Joint Conference on Neural Networks, vol. 2, pp. 425-430 (1989)

(9) B. L. Lu, H. Kita \& Y. Nishikawa: "Inversion of feedforward neural networks by a separable programming", Proc. of World Congress on Neural Networks, vol. 4, pp. 415-420(1993)

(10) B. L. Lu: "Architectures, learning and inversion algorithms for multilayer neural nctworks", Ph. D. thesis, Dept. of Electrical Engineering, Iyoto University (1094)

(11) S. Lee \& R. M. Kil : "Inverse mapping of continuous functions using local and global information", IEEE Trans. Neural Networks, vol. 5, no. 3. pp. 409-423 (1994)

(12) C. E. Miller: "The simplex method for local separable programming", in R. L. Graves \& P. Wolfe (Eds.), Recent Advances in Mathematical Programming, McGraw-Hill, pp. 89-100 (1963)

(13) M. S. Bazaraa, H. D. Sherali \& C. IV. Shetty: Nonlinear Programming Theory and Algorithms, 2nd Ed., Jonn Wilcy and Sons (1903)

(14) D. E. Rumclhart \& J. L. McClelland: Parallel Distributed Processing: Explorations in the Microstructure of Cognition, vol. 1, The MIT Press (1986)

\section{Appendix}

The following notation is used for clescribing the inversion algorithm for multilayer neural networks.

$N_{k}$ : number of units in the layer $k, 1 \leq k \leq 3$,

$x_{k j}$ : output of the $j$ th unit in the layer $h$, where $x_{1 j}$ denotes the input of the $j$ th unit,

$\vec{x}_{k j}$ : prescribed output of the $j$ th unit in the layer $k$,

$b_{k j}: \quad$ total input to the $j$ th unit in the layer $k$,

$w_{k j i}$ : woight connecting the $i$ th wnit in the layer 
$(k-1)$ to the $j$ th unit in the layer $k$, bias $_{k j}$ : bias of the $j$ th unit in the layer $k$, $f: \quad$ sigmoid activation function, $f(z)=1 /(1+\exp (-z))$,

$f^{-1}$ : inverse of the sigmoid activation function, $f^{-1}(z)=\ln (z /(1-z))$,

$\left[\gamma_{j}, \theta_{j}\right]$ : computing range of the inversion of the $j$ th unit in the input layer.

Bao-Liang Lu (Non-member) He received the

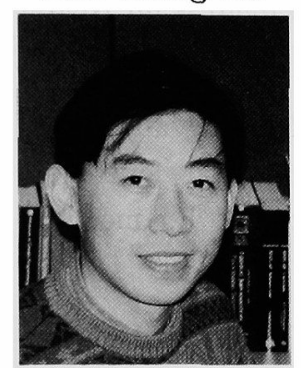
B. S. degree in Automation and Instrument Engineering from Qingdao Institute of Chemical Technology, Qingdao, China in 1982, the M. S. degree in Computer Science from Northwestern Polytechnical University, Xi'an, China in 1989, and the Dr. of Eng. degree in Electrical Engineering from Iiyoto University, Kyoto, Japan in 1994. He was a Research Assistant at Qingdao Institute of Chemical Technology from 1982 to 1986. He joined the BioMimetic Control Research Center at the Institute of Physical and Chemical Research (RIKEN) in April 1994. His research interest includes neural networks, machine learning, and robotics. He is a member of JNNS, IEICE, and IEEE.
Koji Ito

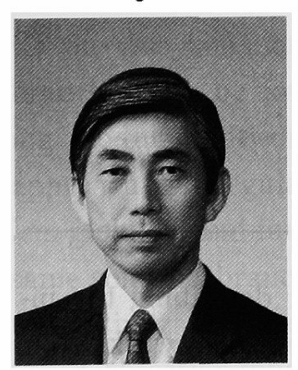

(Member) He was born in 1944.

He received the B. S. degree from Nagoya Institute of Technology in 1967 , and the M. S. degree in 1969 and the Dr. of Eng. degree in 1976 from Nagoya University. From 1970, he was a Research Assistant at Automatic Control Lab., Faculty of Engineering,

Nagoya University. From 1979, he was an Associate Professor in the Department of Computer and System Engineering at Hiroshima University. Since 1992, he has been a professor in the Department of Information and Computer Sciences at Toyohashi University of Technology. Since 1993, he has also held an additional post of head, Lab. for Bio-Mimetic Control Systems at RIIEN: Bio-Mimetic Control Research Center. His main research interests are in the design and control of robotics and prostheses, and computational neural sciences, in particular, biological motor control. He is a. member of IEEE, SICE, and IEICE. 